\title{
Multicenter, Open-Label, Two-Arm, Pilot Trial for Safety Reduction of Basal Insulin Dose Combined with SGLT2 Inhibitor in Type 1 Diabetes Mellitus: Study Protocol for RISING-STAR.
}

Masahide Hamaguchi ( $\nabla$ mhama@koto.kpu-m.ac.jp )

Kyoto Prefectural University of Medicine, Graduate School of Medical Science https://orcid.org/00000002-8651-4445

Yoshitaka Hashimoto

Kyoto Prefectural University of Medicine

Toru Tanaka Japanese Red Cross Kyoto Daiichi Hospital

Goji Hasegawa

Japanese Red Cross Kyoto Daini Hospital

Michiyo Ishii

Otsu City Hospital

Hiroshi Okada

Matsushita Memorial Hospital

Kazuteru Mitsuhashi

Fukuchiyama City Hospital

Noriyuki Kitagawa

Kameoka Municipal Hospital

Emi Ushigome

Kyoto Prefectural University of Medicine

Masahiro Yamazaki

Kyoto Prefectural University of Medicine

Michiaki Fukui

Kyoto Prefectural University of Medicine

\section{Study Protocol}

Keywords: type 1 diabetes mellitus, SGLT2 inhibitor, insulin dose, hypoglycemia, ketone body, ketosis, ketoacidosis 
DOI: https://doi.org/10.21203/rs.3.rs-52292/v1

License: (c) (1) This work is licensed under a Creative Commons Attribution 4.0 International License. Read Full License 


\section{Abstract}

Background: SGLT2 inhibitor combined with insulin therapy is a novel therapy for patients with type 1 diabetes mellitus. Without the reduction of basal insulin, hypoglycemia could occur frequently in this therapy. But diabetic ketoacidosis is an undesirable adverse effect in case with basal insulin reduction. The aim of this study is to explore whether the reduction of the basal insulin dose combined with SGLT2 inhibitor in patients with type 1 diabetes mellitus can reduce the frequency of hypoglycemia and be used safely. We hypothesized that with an adequate basal insulin dose, the frequency of hypoglycemia is higher if the basal insulin dose is not reduced when combined with SGLT2 inhibitor.

Methods and Analysis: The study has a two-arm design; 60 subjects with type 1 diabetes mellitus are being recruited from 7 hospitals. The basal insulin dose before the start of the SGLT2 inhibitor combination therapy is the reference. Study subjects are stratified into two groups based on the ratio of basal insulin daily dose (Basal) to total daily insulin dose (TDD). The subjects are instructed to reduce the basal insulin dose by $10 \%$ or $0 \%$ for Basal to TDD ratio of $<0.4$ and $\geq 0.4$, respectively.

The primary outcome is the frequency of hypoglycemia per day during the intervention period (administration of SGLT2 inhibitor) as determined by self-monitoring of blood glucose (SMBG). The secondary outcome is the frequency of ketosis before and after the intervention.

Discussion: $10 \%$ basal insulin reduction could reduce hypoglycemia as well as could not increase ketosis in case that the ratio of basal insulin daily dose to total daily insulin dose is 0.4 or higher, which improve the efficacy and safety of SGLT2 inhibitor treatment patients with type 1 diabetes mellitus.

Ethics and Dissemination: The study was approved by Kyoto Prefectural University of Medicine, Clinical Research Review Board (CRB5180001). The results will be disseminated through presentations at appropriate conferences and meetings, and published in peer-reviewed journals.

Trial registration: Registered with Japan Registry of Clinical Trials (jRCTs051190114) on 2 March, 2020. https://rctportal.niph.go.jp/detail/jr?trial_id=jRCTs051190114)

\section{Background}

Type 1 diabetes mellitus (T1DM) is a disorder characterized by absolute insulin deficiency, mainly due to autoimmune-mediated pancreatic $\beta$-cell destruction. Although the cause of pancreatic $\beta$-cell destruction is not completely elucidated, susceptibility genes and environmental factors have been implicated [1]. The number of patients with T1DM with absolute insulin deficiency is estimated at approximately $100,000-140,000$ in Japan. The age of onset is mainly in childhood to adolescence, and in Japan, the incidence rate in persons aged $0-19$ years is $4.4 / 1000$ [2].

In Japan, oral diabetic medication is not covered by the national health insurance program, making intensive insulin therapy the principal treatment for T1DM. The insulin dose should be self-titrated based 
on the quantity of carbohydrate ingested. Therefore, therapy with only insulin may cause increased body weight, especially in T1DM patients with excess carbohydrate intake, which increases the risk of macrovascular complications. On the contrary, although low-carbohydrate diets can reduce the total insulin dose and the number of self-injections, they could result in nutritional imbalances. Increased mean body mass index (BMI) reported in T1DM patients [3] additionally increases the risk of cardiovascular disorders.

Sodium-glucose co-transporter (SGLT)-2 inhibitors reduce hyperglycemia via insulin-independent mechanisms by increasing glucose elimination via the kidneys [4]. Although oral anti-diabetic drugs that do not affect insulin secretion have been thought to be effective in the treatment of T1DM and in the prevention of diabetic complications, they have not previously been approved for T1DM. Recently, an SGLT2 inhibitor, dapagliflozin, in combination with insulin therapy, was approved for T1DM [4]. Dapagliflozin selectively inhibits SGLT2 located in the proximal renal tubule, which promotes the excretion of glucose in urine by suppressing the reabsorption of glucose. This action lowers plasma glucose concentration. Since the plasma glucose lowering effect is insulin-independent, SGLT2 inhibitors cause hypoglycemia less frequently, and lead to body weight loss in T1DM patients [5]. In addition, since SGLT2 inhibitors prevent cardiovascular complications in patients with type 2 diabetes mellitus [6], these benefits are also expected in T1DM patients [7].

Hypoglycemia is one of the important determinants of glycemic control in the treatment of T1DM [8]. Many patients with T1DM struggle to achieve glycemic control and experience significant fluctuations in blood glucose levels despite insulin treatment $[4,9]$. Across randomized trials, SGLT2 inhibitors demonstrated significant reductions in glycated hemoglobin, glucose exposure, and measures of glycemic variability, as well as increased time in the target glycemic range when administered as an adjunct to insulin [4].

To prevent hypoglycemia, many T1DM patients might reduce the basal insulin dose, which might impact sugar control. While the addition of SGLT2 inhibitors might reduce the risk of hypoglycemia, their use has been reported to increase the frequency of diabetic ketoacidosis [10]. Therefore, the reduction of the insulin dose to prevent hypoglycemia during adjunctive SGLT2 inhibitor treatment may increase the risk of ketoacidosis, whereas maintaining the same insulin dose in order to prevent ketoacidosis may increase the risk of hypoglycemia. An algorithm for insulin adjustment would be beneficial when combining SGLT2 inhibitor treatment with insulin in patients with type 1 diabetes mellitus. We therefore aim to explore whether the reduction of the basal insulin dose combined with SGLT2 inhibitor in patients with T1DM can reduce the frequency of hypoglycemia. We hypothesize that with an adequate basal insulin dose, the frequency of hypoglycemia will be higher if the basal insulin dose is not reduced when combined with the SGLT2 inhibitor.

\section{Methods And Analysis}

\section{Study protocol}


The study is a multi-center, open-label, non-randomized, exploratory, prospective, interventional study (Figure 1). The subjects are stratified into two groups based on the ratio of basal insulin (Basal) to the total daily insulin dose (TDD) (Basal/TDD, <0.4 or $\geq 0.4$ ). Instructions to the study subjects are found in Table 1.

A pre-observation period of 4 weeks is set before the intervention (administration of SGLT2 inhibitor). During this period, study subjects will measure and record fasting plasma beta-hydroxybutyric acid (BOHB), flash glucose monitoring (FGM), and self-monitoring of blood glucose (SMBG). The start of SGLT2 inhibitor administration is set as day zero of the observation period. The study subjects will visit the research institutions 4 times; at weeks $-4,0,2$, and 4 . The study subjects were given a digital camera and instructed to take a picture of each meal they eat throughout the observation period.

[Group A] Study subjects reduce basal insulin dose by $0 \%$ (instructed to reduce bolus insulin dose)

Subjects whose Basal/TDD ratio is $<0.4$ are instructed not to reduce basal insulin dose but to reduce bolus insulin dose by $10 \%$. The $10 \%$ reduction of the bolus insulin dose is based on $90 \%$ carbohydrate insulin ratio (CIR), and is rounded to the nearest whole number in case of insulin therapy with multiple daily injections (MDI), or rounded down to two decimal places in case of continuous subcutaneous insulin infusion (CSII). The study subjects are instructed to follow this instruction for 3 days from the start of the intervention. After the fourth day, subjects can titrate both basal and bolus dose according to "Algorithm for Basal Insulin Titration after SGLT2 administration (Figure 2)" and "Algorithm for Bolus Insulin Titration after SGLT2 administration (Figure 3)."

[Group B] The study subjects are instructed to reduce basal insulin by $10 \%$

Study subjects whose Basal/TDD is $\geq 0.4$ are instructed to reduce total insulin dose by $10 \%$, reducing the basal insulin dose only. The dose of basal insulin is rounded down to one decimal places in case of MDI or to two decimal places in case of CSII. The study subjects are instructed to follow this instruction for 3 days from the start of the intervention. After the fourth day, subjects can titrate both basal and bolus dose according to "Algorithm for Basal Insulin Titration after SGLT2 administration (Figure 2)" and "Algorithm for Bolus Insulin Titration after SGLT2 administration (Figure 3)."

\section{Eligibility Criteria}

In line with the objectives of the study and for ensuring the safety of the subjects, the inclusion and exclusion criteria explicated in table 2 were set. In order to appropriately evaluate the efficacy of the study drugs, patients who require a legal representative are excluded.

\section{Observations}

Observations and the schedule are shown in table 3 and table 4. In principle, the study subjects will visit the research institutions, and at every visit, blood tests (fasting) and urine tests (spot) is performed. Investigators collect and enter the results of examinations listed in table 3 in the case report form (CRF), 
and send the CRF to the data center. Adverse events are followed as safety endpoints throughout the study. The items which the study subjects themselves measure are recorded onto the specific documents and sent to the data center via the investigators.

\section{Data management and monitoring}

Data collection and management are carried out by third-party entities to avoid bias. The data management is performed by Soiken Inc. Data Management Group (the Data Centre). The Data Centre prepared a procedure manual for data management. The Data Centre's approval is required prior to sending any data related to the subjects in an electronic format. If data is transmitted over an unsecured electronic network, the data must be encoded at the source. Linkable anonymization by central registration number is used to identify the subjects. The investigators are responsible for appropriate storage of the correspondence table prepared by them to identify the subjects, in accordance with the procedures at the particular research institution. This correspondence table will be retained for 5 years after the completion of this study. Appropriate measures such as encoding or deletion are taken to ensure that the subjects cannot be identified, in accordance with applicable laws and regulations.

The Data Centre monitors this study to manage and ensure quality. The monitoring manager monitors the subjects in accordance with the prescribed manual on monitoring. For data quality management, the principal investigator and Central Committee confirms the progress of this study as necessary through the Data Centre to ensure conformance with the protocol and the Ethical Guidelines for Medical and Health Research Involving Human Subjects (28 February 2017; Ministry of Education, Culture, Sports, Science and Technology/Ministry of Health, Labor and Welfare) and the Clinical Trials Act (14 April 2017; Ministry of Education, Culture, Sports, Science and Technology/Ministry of Health, Labor and Welfare).

\section{Primary endpoints}

Frequency of hypoglycemia per day confirmed by self-monitoring of blood glucose (SMBG) during the intervention period (administration of SGLT2 inhibitor) (number of hypoglycemia/day).

\section{Secondary endpoints}

1. Frequency of ketosis before and after the intervention

Ketosis is defined as plasma $B O H B \geqq 600 \mu \mathrm{mol} / \mathrm{L}[11-12]$. Frequency of ketosis is defined as the proportion of the number of days in which ketosis occurs in the study subjects during the pre-observation period and the observation period after the intervention.

2. Frequency of hypoglycemia per day before and after the intervention detected by FGM (number of hypoglycemia/day)

3. Change and difference in change between the intervention groups on the following items, before and after the intervention 
3-1. Fasting plasma beta-hydroxybutyric acid (ketone body)

3-2. Basal insulin dose*, bolus insulin dose*, total insulin dose*, Carbohydrate Insulin Ratio (CIR), and Insulin Sensitivity Factor (ISF)

3-3. Time spent in hypoglycemia (percentage of time spent in glucose range $\leqq 70 \mathrm{mg} / \mathrm{dl}$ ), time in hyperglycemia (percentage of time spent in glucose range $\geqq 70 \mathrm{mg} / \mathrm{dl}$ ) time spent with nocturnal hypoglycemia, detected by FGM

3-4. Vital signs: blood pressure, pulse rate

3-5. Body weight, BMI, skeletal muscle masst, fat mass $†$

3-6. Blood biomarkers: red blood cell count, white blood cell count, hemoglobin, hematocrit, blood platelet count, hepatic enzymes (AST, ALT, LDH, ALP, gamma-GTP), UA, T-Chol, HDL, LDL, TG, BUN, Cre, eGFR, BNPt, HbA1c (or glycoalbumin), plasma glucose, albumin, CPR + , and CPR index $\dagger$

3-7. Urine biomarker: specific gravity, $\mathrm{pH}$, protein, glucose, ketone body, occult blood, urobilinogen, bilirubin, microalbumin, creatinine, and microalbumin-creatinine ratio

3-8. Total ketone body $\dagger$, beta-hydroxybutyric acidt, and acetoacetic acid $\dagger$

3-9. QOL score (DTSQ)

*measured by daily dose, tmeasured at visit 2 and 4 only

\section{Safety endpoints}

Frequency of adverse events (including frequency of hypoglycemia detected by FGM)

\section{Exploratory endpoints}

Correlation between change in dietary habit, dietary content, and nutrient intake and change in fasting plasma beta-hydroxybutyric acid.

Change in plasma microRNA profile.

Change in cardiac function estimated by ultrasonic echocardiography (UCG).

\section{Rationale underlying the sample size}

This study is designed as an exploratory study, and no prior studies have reported the frequency of hypoglycemia after the administration of SGLT2 inhibitor. We hypothesized that the frequency of hypoglycemia per day would increase after the administration of SGLT2 inhibitor, if the dose of basal insulin was not titrated. The frequency of hypoglycemia has been reported to be $7 \pm 6$ times/month when insulin glargine is used in patients with TIDM [13]. The assumed increased hypoglycemia, and the 
required sample sizes are plotted in figure 4 . When 26 patients were in one group, the \% increase in hypoglycemia was more than $60 \%$, which was deemed adequate. With an estimated dropout rate of $10 \%$, the planned number of subjects (60 subjects, 30 in each group) is thought to have enough statistical power for an increased hypoglycemia frequency of more than $60 \%$ (11.2 \pm 6 times/month) in Group A (Basal/TDD is $<0.4$, the study subjects are instructed to reduce basal insulin dose $0 \%$ but instructed to reduce bolus insulin dose 10\%) from $7 \pm 6$ times/month in Group B (Basal/TDD is 0.4 or higher, the study subjects are instructed to reduce basal insulin 10\%).

This study is being conducted at 7 research institutions where a total of 350 patients with T1DM are being treated. From a previous survey, $30 \%$ of these patients are eligible to use SGLT2 inhibitors as per the Japanese labelled indications, meaning that approximately 100 patients could potentially use the SGLT2 inhibitor with insulin. Among the 100 patients, all patients who sign a written consent form are enrolled in the study, and stratified into Group A (Basal/TDD is $<0.4$, subjects instructed to reduce basal insulin dose $0 \%$ and bolus insulin dose by $10 \%$ ) or Group B (Basal/TDD $\geq 0.4$, subjects instructed to reduce basal insulin by $10 \%$ ). The proportion of patients who will consent is assumed to be $80 \%$, and the proportion of patients who meet the inclusion criteria and none of the exclusion criteria is assumed to be $80 \%$. Under these conditions, the target number of 60 study subjects is feasible.

\section{Basic analytical procedure}

For primary and secondary endpoints, both full analysis set (FAS) and per protocol set (PPS) will be analyzed. The analyses will be conducted with a two-sided significance level of $5 \%$. A biomedical statistic expert created a statistical analysis plan (SAP) separately, and specified the details of the statistical methods including handling data. The SAP was prepared before the database lock.

When amendment of the SAP is needed, the data handling committee will carefully consider the effect of the amendment on the study itself, the results of the study, the ethical concerns and scientific validity of the effect. If the data handling committee judges that the amendment of the SAP is valid, the procedure, the content, and the result of the discussion is recorded.

\section{Analysis of study subjects' background information}

Summary statistics will be calculated for background information of study subjects per group.

Frequencies and percentages of categories are shown for nominal variables. Summary statistics (number of cases, mean, standard deviation, minimum, median, and maximum values) will be calculated for continuous variables. For comparisons between the groups, Chi-square test will be used for nominal variables. If the proportion of the cells with expected frequency of less than 5 is $20 \%$ or more, Fisher's exact test will be used. For comparisons between groups for continuous variables, two-sample t-test or Wilcoxon rank sum test is used.

\section{Analysis of the primary endpoint}


Summary statistics will be calculated for the number of hypoglycemic events per day (plasma glucose level defined by $S M B G \leq 70 \mathrm{mg} / \mathrm{dL}$ ) during the intervention period (from baseline to week 4) using FAS as main analysis set and PPS for sensibility analysis set. For comparisons between groups, two-sample ttest and analysis of covariance will be conducted, and differences between the groups and their $95 \%$ confidence intervals calculated. $\mathrm{HbA} 1 \mathrm{c}$, age, and frequency of hypoglycemia ( $\leq 70 \mathrm{mg} / \mathrm{dL}$, confirmed by SMBG) per day during the pre-observation period before the intervention (week -4 to baseline) will be used as covariates in the analysis of covariance. If the data distribution does not follow a normal distribution, summary statistics will be calculated after logarithmic transformation.

\section{Analysis of the secondary endpoints}

For the analysis of the secondary endpoint 1 (frequency of ketosis) and 2 (frequency of hypoglycemia detected by FGM), summary statistics of measurements and changes will be calculated using FAS during the pre-observation period before the intervention (week -4 to baseline) and the observation period after the intervention (baseline to week 4 ) in each group. For the measurements, two-sample t-test will be used for comparisons between groups, and for the change, one-sample t-test will be used for comparison in each group. If the data distribution does not follow a normal distribution, summary statistics will be calculated after logarithmic transformation.

For the analysis of the secondary endpoint 3, summary statistics will be calculated using FAS for measurements at each observation point and change in the measurements from baseline to each observation point after the intervention. For the measurement, two-sample t-test will be used for comparisons between groups, and for the change in the measurement, one-sample t-test will be used for comparison in each group.

\section{Analysis of the safety endpoints}

For the analysis of the safety endpoints, a table of all adverse events and diseases or the like will be created for each group using the safety analysis set, and comparisons performed between groups as necessary using Fisher's exact test.

\section{Analysis of exploratory endpoints}

For the analysis or correlation between change in dietary habit, dietary content, and nutrient intake and change in fasting plasma BOHB acid, Spearman rank correlation coefficient and its $95 \%$ confidence interval will be calculated, and evaluated for significance. The subjects have been instructed to take pictures of each of their meals using digital camera. The stored images will be uploaded to a cloud, and diabetologists will analyze the images according to the Standard Tables of Food Composition in Japan using a specialized application (Asken, Wit Co., Ltd.). The volumes and calories of carbohydrates, proteins, fats, and nutrient intakes will be calculated by the system referred to as "online nutritional evaluation."

\section{Ethics and Dissemination}


The protocol was approved by Kyoto Prefectural University of Medicine, Clinical Research Review Board (CRB5180001), and registered at jRCT (jRCTs051190114).

The findings will provide knowledge about the reduction of the basal insulin dose combined with an SGLT2 inhibitor in patients with T1DM, which may reduce the frequency of hypoglycemia associated with the combination therapy. The results will be disseminated through presentations at appropriate conferences and meetings, and publications in peer-reviewed journals.

All parties involved in this study will endeavor to protect the personal information of study subjects. This study is being conducted in accordance with the Personal Information Protection Law, and other legislation/law and regulations. Unique information (initial, carte number) of study subjects is stored securely in the research institutions, and information that allows a person outside the research institution to identify the study subjects (such as name, address, telephone number, etc.) is not included in CRFs and registration database.

A central registration number is used when the data center enquires regarding data from a study subject at a research institution. Investigators use the correspondence tables to identify their research subjects (anonymization), which are managed by themselves. Investigators store the correspondence tables securely and keep them properly until at least 5 years from the day of the completion of the study. The person responsible for personal information management at each research institution shall be the responsible investigator unless otherwise specified.

The principal investigator, the responsible investigators, sub-investigators, and the heads of the research institutions agree to store the study-related information properly until at least 5 years from the completion date of the study. The responsible person in charge of data management also stores the study-related information such as CRF as source documents and electronic information, such as data sets, are properly stored in the same way.

After the storage period, the data is to be discarded. Anonymized data collected for the analysis is kept stored for future secondary study such as meta-analysis. If the anonymized data is used for other studies, approval from the ethics review board is required before study implementation.

Samples for special blood tests are measured in laboratories utilized by each research institution. After the requisite data is obtained, the samples are disposed of, in accordance with standard procedural guidelines.

The study explores whether the reduction of the basal insulin dose combined with SGLT2 inhibitor in patients with type 1 diabetes mellitus can reduce the frequency of hypoglycemia with an overall acceptable safety profile.

The treatment intervention in this study is based on the Basal/TDD at consent. Therefore, the study subjects cannot always receive their desired treatment. However, all study subjects will receive regular medication, and will measure ketone body and blood glucose, and titrate both basal and bolus insulin 
after the fourth day of the intervention. As a result, the study subjects may receive better medical care than would be the case if they did not participate in this study.

To reduce the burden on study subjects, the change of medications during the study are not restricted. Special blood tests are conducted using the residual samples to reduce the burden caused by multiple blood draws. Therefore, generally, special compensation is not provided even when health damages are considered to be caused by the drug(s) used in the study. For reporting purposes, all incidences during the study will be treated similarly to health damage or medical accidents caused by regular medication. The compensation in such a case is based on the Adverse Drug Reaction Relief System of the Pharmaceutical and Medical Devices Agency, Product Liability Law, or product liability insurance. The principle investigator contracted the insurance for clinical study corresponding to any incidences. The current research is carried out in accordance with the Clinical Trial Act and other laws and regulations. Burdens and predicted risks are minimized as much as possible from the perspective of protecting human subjects. In particular, we believe that the risk of adverse effects can be minimized by discontinuing the study treatment as soon as a symptom emerges and providing treatments using appropriate procedures. Based on the overall evaluation, and taking into account conceivable benefits from the study participation, this study protocol is considered appropriate to implement and adequate in terms of ethical considerations.

\section{Discussion}

This study will clarify the efficacy and safety of SGLT2 inhibitor treatment in patients with type 1 diabetes mellitus (T1DM).

The dose of insulin should be self-titrated based on the quantity of carbohydrate ingested. Therefore, therapy with only insulin may cause increased body weight, especially in T1DM patients with excess carbohydrate intake, which increases the risk of macrovascular complications. On the contrary, although low-carbohydrate diets can reduce the total insulin dose and the number of self-injections, it could result in nutritional imbalances. Increased mean body mass index (BMI) reported in T1DM patients [3] additionally increases the risk of cardiovascular disorders.

Recently, a SGLT2 inhibitor, dapagliflozin, was approved for T1DM when combined with insulin therapy. SGLT2 inhibitors cause hypoglycemia less frequently, and leads to body weight loss in patients with type 2 diabetes mellitus [5]. In addition, since SGLT2 inhibitors prevents cardiovascular complications in patients with type 2 diabetes mellitus [6], the beneficial effects of the SGLT2 inhibitors are also expected in patients with T1DM [7].

Hypoglycemia is one of the important factors that affects glycemic control in the treatment of T1DM. While addition of SGLT2 inhibitors might reduce the risk of hypoglycemia, their use has been reported to increase the frequency of diabetic ketoacidosis [8]. Therefore, reduction of insulin dose to prevent hypoglycemia during adjunctive SGLT2 inhibitor treatment may increase the risk of ketoacidosis whereas maintained insulin dose in this setting to prevent ketoacidosis may increase the risk of hypoglycemia. 
Therefore, an algorithm for insulin adjustment would be beneficial when combining SGLT2 inhibitor treatment with insulin in patients with type 1 diabetes mellitus.

This study will be clarify effective and safe ways to introduce SGLT2 inhibitors in patients with type 1 diabetes.

\section{Declarations}

\section{Ethics approval and consent to participate}

This study was registered with the Japan Registry of Clinical Trials (jRCTs051190114) and has been approved by the ethics committees of the Kyoto Prefectural University of Medicine (CRB5180001). This study is to be conducted according to the Declaration of Helsinki. Written informed consent will be obtained from all the participants.

\section{Consent for publication}

Not applicable

\section{Funding}

This study, including the article processing charge, is funded by AstraZeneca K.K. and Ono Pharmaceutical Co., Ltd. No drugs will be donated or funded by the sponsor. The funding bodies had no role in study design, data collection and analysis, decision to publish, or preparation of the manuscript.

\section{Competing interests}

1. H. reports grants from Asahi Kasei Pharma, Nippon Boehringer Ingelheim Co. Ltd., Mitsubishi Tanabe Pharma Corp., Daiichi Sankyo Co. Ltd., Sanofi K.K., Takeda Pharma Co. Ltd, Astellas Pharma Inc., Kyowa Kirin Co. Ltd., Sumitomo Dainippon Pharma Co. Ltd., Novo Nordisk Pharma Ltd., and Eli Lilly Japan K.K., outside the submitted work. M. A. reports personal fees from Novo Nordisk Pharma Ltd., Abbott Japan Co. Ltd., AstraZeneca plc, Kowa Pharma Co. Ltd., Ono Pharma Co. Ltd., Sumitomo Dainippon Pharma Co. Ltd., and Takeda Pharma Co. Ltd. outside the submitted work. M. Y. reports personal fees from MSD K.K., Sumitomo Dainippon Pharma Co. Ltd., Kowa Pharma Co. Ltd., AstraZeneca plc., Takeda Pharma Co. Ltd, Kyowa Kirin Co. Ltd., Daiichi Sankyo Co. Ltd., Kowa Pharma Co. Ltd., and Ono Phama Co., Ltd. outside the submitted work.

2. H. reports grant from Asahi Kasei Pharma and personal fees from Daiichi Sankyo Co. Ltd., Mitsubishi Tanabe Pharma Corp., Sanofi K.K., and Novo Nordisk Pharma Ltd., outside the submitted work.

3. F. received grants from Nippon Boehringer Ingelheim Co. Ltd., Kissei Phama Co. Ltd., Mitsubishi Tanabe Pharma Corp, Daiichi Sankyo Co. Ltd., Sanofi K.K., Takeda Pharma Co. Ltd., Astellas Pharma Inc., MSD K.K., Kyowa Kirin Co., Ltd., Sumitomo Dainippon Pharma Co., Ltd., Kowa Pharma Co. Ltd., Novo Nordisk Pharma Ltd., Ono Pharma Co. Ltd., Sanwa Kagagu Kenkyusho CO., Ltd., Eli Lilly Japan 
K.K., Taisho Pharma Co., Ltd., Terumo Corp., Tejin Pharma Ltd., Nippon Chemiphar Co., Ltd., Abbott japan Co. Ltd., and Johnson \& Johnson k.k. Medical Co., and received honoraria from Nippon Boehringer Ingelheim Co., Ltd., Kissei Pharma Co., Ltd., Mitsubishi Tanabe Pharma Corp., Daiichi Sankyo Co. Ltd., Sanofi K.K., Takeda Pharma Co. Ltd., Astellas Pharma Inc., MSD K.K., Kyowa Kirin Co. Ltd., Sumitomo Dainippon Pharma Co. Ltd., Kowa Pharma Co. Ltd., Novo Nordisk Pharma Ltd., Ono Pharma Co. Ltd., Sanwa Kagaku Kenkyusho Co. Ltd., Eli Lilly Japan K.K., Taisho Pharma Co., Ltd., Bayer Yakuhin, Ltd., AstraZeneca K.K., Mochida Pharma Co. Ltd., Abbott japan Co. Ltd., Teijin Pharma Ltd., Arkray Inc., Medtronic Japan Co. Ltd., and Nipro Corp. outside the submitted work. The other authors have nothing to disclose.

\section{Availability of data and materials}

Data sharing is not applicable to this article as no datasets were generated or analyzed during the current study.

\section{Authors' contributions}

$\mathrm{MH}$ led the drafting of the manuscript. YH and MF reviewed the manuscript and study design and contributed to the final draft. The other authors will recruit participants and contributed to the final draft.

\section{Patient and Public Involvement statement}

Patients have not been involved in the design of the study, the selection of research questions, or outcome measurements. Participants will not be involved in the interpretation or the write-up of results. Participants will be given a simple summary of the study outcomes, written in Japanese, once the study has been completed.

Acknowledgments: We would like to thank Editage (www.editage.com) for English language editing. The authors thank all the clinical staff for their assistance with the execution of the clinical trial, and Soiken Inc. for their technical assistance in the launch and execution of this trial.

\section{Trial status}

This study started on 2 March, 2020 with protocol version 1.1. The recruitment will be completed at 31 August 2020.

\section{References}

1. Ministry of Health, Labor and Welfare. Scientific Research Grant 2017, Study of type 1 diabetes mellitus to estimate actual condition, objective diagnostic criteria, and severity assessment based on daily activity and social activity (2018) Type 1 diabetes mellitus in which insulin secretion is completely lost. http://dmic.ncgm.go.jp/content/type1_insulin_20180817.pdf. Accessed 18 July 2019. 
2. International Diabetic Federation. Diabetes Atlas Eighth Edition. 2017. https://www.idf.org/elibrary/epidemiology-research/diabetes-atlas.html. Accessed 18 July 2019.

3. Japan Diabetes Clinical Data Management Study Group. http://jddm.jp/data/index-2017.html (2007). Accessed 18 July 2019.

4. Boeder S, Edelman SV. Sodium-glucose Co-Transporter Inhibitors as Adjunctive Treatment to Insulin in Type 1 Diabetes: A Review of Randomized Controlled Trials. Diabetes Obes Metab. 2019;21(Suppl 2(Suppl 2):62-77.

5. Nauck MA, Del Prato S, Meier JJ, Duran-Garcia S, Rohwedder K, ELze M, et al. Dapagliflozin Versus Glipizide as Add-on Therapy in Patients With Type 2 Diabetes Who Have Inadequate Glycemic Control With Metformin A randomized, 52-week, double-blind, active-controlled noninferiority trial. Diabetes Care. 2011;34:2015-22.

6. Wanner C, Heerspink HJL, Zinman B, Inzucchi SE, Koitka-Weber A, Matheus M, et al. Empagliflozin and Kidney Function Decline in Patients with Type 2 Diabetes: A Slope Analysis from the EMPA-REG OUTCOME Trial. J Am Soc Nephrol. 2018;29:2755-69.

7. Dandona P, Mathieu C, Phillip M, Hansen L, Griffen SC, Tschope D, et al. Efficacy and safety of dapagliflozin in patients with inadequately controlled type 1 diabetes (DEPICT-1): 24 week results from a multicenter, double-blind, phase 3, randomized controlled trial. Lancet Diabetes Endocrinol. 2017;5:864-76.

8. Lehecka KE, Renukuntla VS, Heptulla RA. Insight Into Hypoglycemia in Pediatric Type 1 Diabetes Mellitus. Int J Pediatr Endocrinol. 2012;2012(1):19.

9. Kovatchev B, Cobelli C. Glucose Variability: Timing, Risk Analysis, and Relationship to Hypoglycemia in Diabetes. Diabetes Care. 2016;39(4):502-10.

10. Rosenstock J, Marquard J, Laffel LM, Neubacher D, Kaspers S, Cherney DZ, et al. Empagliflozin as Adjunctive to Insulin Therapy in Type 1 Diabetes: The EASE Trials. Diabetes Care. 2018;41(12):256069.

11. Schade DS, Eaton RP. (1982) Metabolic and clinical significance of ketosis. Spec Top in Endocrinol Metab. 1982;4:1-27.

12. Wiggam MI, O'Kane MJ, Harper R, Atkinson AB, Hadden DR, Trimble ER, et al. Treatment of diabetic ketoacidosis using normalization of blood 3-hydroxybutyrate concentration as the endpoint of emergency management. A randomized controlled study. Diabetes Care. 1997;20(9):1347-52.

13. Urakami T, Kuwabara R, Habu M, Okuno M, Suzuki J, Takahashi S. Efficacy and safety of switching to insulin glulisine from other rapid-acting insulin analogs in children with type 1 diabetes. $J$ Diabetes Investig. 2015;6(1):87-90.

\section{Tables}




\begin{tabular}{|c|c|c|}
\hline & \multicolumn{2}{|c|}{ Insulin self-titration according to the algorithm } \\
\hline 2 & \multicolumn{2}{|c|}{ Recommendation to drink water } \\
\hline & (a) & $\begin{array}{l}\text { Recommend to drink } 1.5 \mathrm{~L} / \text { day of water, if the study subjects do not have chronic renal } \\
\text { failure or chronic heart failure. }\end{array}$ \\
\hline & (b) & $\begin{array}{l}\text { If the study subjects have chronic renal failure or chronic heart failure, the attending } \\
\text { physician will instruct on suitable volume of fluid intake according to the condition. }\end{array}$ \\
\hline 3 & \multicolumn{2}{|r|}{ Symptoms that could be indicative of ketoacidosis } \\
\hline & (a) & measure plasma beta-hydroxybutyric acid \\
\hline & (b) & Consult the attending physician if the plasma beta-hydroxybutyric acid is $\geq 600 \mu \mathrm{mol} / \mathrm{L}$ \\
\hline
\end{tabular}

4 Symptoms that could be indicative of sick day

(a) consider ketoacidosis even if plasma glucose level is normal

(b) measure plasma beta-hydroxybutyric acid every 3-4 hours

(c) intake $30-60 \mathrm{~g}$ of carbohydrate and $200-500 \mathrm{ml}$ of water

(d) inject bolus insulin

(e) avoid excess insulin reduction

(f) call ambulance if abdominal pain, nausea, vomiting, lassitude, respiratory distress, etc. are not improved

5 Criteria for dapagliflozin withdrawal

(a) Consult the attending physician if physical deterioration/illness or sick day

6 Symptoms that require at emergency consultation

symptoms including abdominal pain, nausea, vomiting, las situde, respiratory distress, etc.

(a) are not improved even if plasma glucose, self-measured plasma beta-hydroxybutyric acid is within the normal range.

(b) self-measured plasma beta-hydroxybutyric acid is $1,000 \mu \mathrm{mol} / \mathrm{L}$ or higher, and/or ketoacidosis-related symptoms are not improved

(c) the study subjects brings card to indicate that they are patients with type 1 diabetes mellitus and are administrating SGLT2 inhibitor when receiving emergency consultation 


\section{Table 2. Inclusion and Exclusion Criteria}

\section{Inclusion Criteria}

Patients who meet all of the following criteria may be eligible to be included in this study:

1 Outpatient in the research institutions included in this study, who are diagnosed with type 1 diabetes mellitus before 6 months or more of giving their consent

2 Patients who have conducted intensive insulin therapy for 1 year or longer

3

Patients who are well-educated in carbohydrate counting, and who can conduct insulin self-titration

4 Patients with good understanding of the disease and capability to recognize DKA (symptoms and use of ketone meter)

5 Male and female aged $\geq 20$ years and $<80$ years when giving their consent

6 Patients who provide their consent in a written form

Rationale for the inclusion criteria:

1-3: for the appropriate evaluation of the efficacy outcomes in this study.

4: for the safety of the study subjects in the real-world situation.

5: for the participation of the study subjects by their free will. 


\section{Exclusion Criteria}

3 Patients whose activities of daily living (ADL) are PS 2 or higher

4 Patients with dementia or cognitive impairment

5 Patients whose BMI is less than $18.5 \mathrm{~kg} / \mathrm{m} 2$

6

Patients with a history of ketoacidosis within 3 months before giving their consent

7 Patients with a history of cardiovascular disease (myocardial infarction, heart failure, and angina) within 3 months before giving their consent

8 Patients whose $\mathrm{HbA} 1 \mathrm{c}$ is $10.5 \%$ or higher

9 Patients who had hypoglycemia $\geq 14$ times within 4 weeks of giving their consent

Patients with anemia (male: $\mathrm{Hb}$ is $13 \mathrm{~g} / \mathrm{dL}$ or less, female: $\mathrm{Hb}$ is $12 \mathrm{~g} / \mathrm{dL}$ or less), hypoalbuminemia (serum albumin is $3.5 \mathrm{~g} / \mathrm{dL}$ or less), or

10 nephrotic syndrome (urinary protein is $3.5 \mathrm{~g} /$ day or more, and serum albumin is $\leq 3.0 \mathrm{~g} / \mathrm{dL}$ ) caused by primary diseases other than diabetic nephrop athy

11

Patients who are breastfeeding, pregnant, possibly pregnant, or planning to be pregnant

Patients with neoplasms. A patient who has just undergone chemotherapy

12 and has some early signs of being in remission are be enrolled. However, those who have completed treatment and/or show no relapse can be considered to be participants of this study

13 Patients who have contraindications to using the drug used in the study

14 Patients who have diet therapy with carbohydrate of less than $40 \%$ of total calories

15 Patients with poor adherence as judged by the investigators

Patients with other conditions that the responsible investigator/subinvestigator consider inappropriate to allow participation in the study

\section{Table 3. Observation items}

1. Eligibility information

Observation At consenting, enrollment
point

Observation gender, age/date of birth, inclusion criteria, exclusion criteria, date of giving consent, item total daily insulin dose (TDD), basal insulin dose (Basal), bolus insulin dose, HbA1c

2. Background information 


\section{Observation week -4}

point

Observation height, age, gender, presence/absence of smoking habit, presence/absence of drinking item

habit, wakeup time, bedtime, comorbidity (presence/absence or history of macrovascular/microvascular disease, dyslipidemia, hypertension, hepatic disease), information regarding kinds of medication* (antihypertensives, medicine for dyslipidemia, anti-diabetic agents), type and dose of insulin, allergy

3. physical examination

Observation week -4 , week 0 , week 2 (optional), week 4 point

Observation body temperature, blood pressure (sitting position, office blood pressure), pulse rate, item body weight, BMI*, body composition (skeletal muscle mass, body fat mass)

* obtained by calculation

4. medication information (except study agent and insulin)

Observation point $\quad$ week -4 , week 0 , week 2 (optional), week 4

Observation item presence/absence and content of change in type or dose of medication

5. medication information (study agent, insulin)

\begin{tabular}{|ll|}
\hline Observation point & throughout observation period \\
\hline Observation item & presence/absence of medication of study agent \\
& type and dose of insulin \\
& *the study subjects record onto the study subjects' diary by themselves
\end{tabular}

6. blood tests (fasting)

Observation week -4, week 0 , week 2 (optional), week 4

point

Observation red blood cell count, white blood cell count, hemoglobin, hematocrit, blood platelet

item

count, hepatic enzymes (AST, ALT, LDH, ALP, gamma-GTP), UA, T-Chol, HDL, LDL, TG,

BUN, Cre, eGFR, HbA1c (or glycoalbumin), plasma glucose, serum albumin

Observation week 0 , week 4

point

Observation BNP匹CPR $\triangle \mathrm{CPR}$ index

item

7. urine tests (spot) 
Observation week -4, week 0, week 2 (optional), week 4

point

Observation specific gravity, $\mathrm{pH}$, protein, glucose, ketone body, occult blood, urobilinogen, bilirubin, item u-mAlb, U-Cre, U-mÁlb/Cre ratio

8. special blood tests (fasting, using residual sample of "6. blood tests")

\begin{tabular}{ll}
\hline Observation point & week 0, week 4 \\
Observation item & total ketone body, beta-hydroxybutyric acid, acetoacetic acid, plasma microRNA
\end{tabular}

9. Ultrasound Cardiography (UCG)

Observation week -4, week 4 point

Observation Ultrasound Cardiography (early diastolic filling velocity, atrial filling velocity, E/A, e', item $\quad$ E/e', DT, IVRT, TR, LVEF, LVEDV, LVESV, LAVI, LVDd, LVDs)

10. QOL score (questionnaire to whom the study subjects directly answer)

Observation week -4 , week 0 , week 4

point

Observation DTSQs (The Diabetes Treatment Satisfaction Questionnaire, status version) score.

item DTSQs are questionnaire to measure treatment satisfaction specific to diabetes mellitus, widely used over the world, consists of 8 questions.

11. Other items the study subjects measure by themselves 1 
Observation throughout week -4 to week 0 , week 0 to week 4

point

The study subjects use the Freestyle Libre for FGM after receiving full explanation of how to use it. Since the sensor of the Freestyle Libre can be used for 2 weeks ( 14 days), investigators give 2 sensors to each study subject in advance of each period. The study subject change and install sensor every 2 weeks.

The data which the study subjects measure by themselves are recorded onto the study subjects' diary.

Observation $\otimes$ Fasting plasma beta-hydroxybutyric acid (ketone body)

item

The study subjects measure ketone body once daily before the breakfast at home using Freestyle Libre (Abbott Japan Co., Ltd.) The study subjects also measure the ketone body at their free will at physical deconditioning, sick day or onset of symptoms suggesting ketoacidosis.

$\nabla$ Plasma glucose (FGM)

The study subjects measure the plasma glucose continuously at home using Freestyle Libre (Abbott).

$\rrbracket$ Plasma Glucose (SMBG)

The study subjects conduct SMBG 4 times per day (before breakfast, before lunch, before dinner, and before bedtime) and at the awareness of hypoglycemic symptoms or at FGM values of less than $70 \mathrm{mg} / \mathrm{dL}$ at home using Freestyle (Abbott).

$\nabla$ Dose of insulin

\ Awareness of hypoglycemia

12. Other items the study subjects measure by themselves 2 
Observation throughout observation period

point

Observation $\otimes$ diet and relevant information

item

The study subjects record the diet and relevant information every day throughout the observation period using the application software.

1) gender, age, height, target body weight, activities of daily life: prespecified value (enter once)

2) body weight, percent body fat, body water, alcohol intake, confectionery intake, staple food intake, main dish intake, side dish intake, milk product intake, fruit intake, number of steps (every day)

3) energy, protein, lipid, carbohydrate, dietary fiber, sugar (every meal)

Observation $\otimes$ Fasting plasma beta-hydroxybutyric acid (ketone body)

item

The study subjects measure ketone body once daily before the breakfast at home using Freestyle Libre (Abbott Japan Co., Ltd.) The study subjects also measure the ketone body at their free will at physical deconditioning, sick day or onset of symptoms suggesting ketoacidosis.

$\nabla$ Plasma glucose (FGM)

The study subjects measure the plasma glucose continuously at home using Freestyle Libre (Abbott).

\ Plasma Glucose (SMBG)

The study subjects conduct SMBG 4 times per day (before breakfast, before lunch, before dinner, and before bedtime) and at the awareness of hypoglycemic symptoms or at FGM values of less than $70 \mathrm{mg} / \mathrm{dL}$ at home using Freestyle (Abbott).

\ Dose of insulin

Q Awareness of hypoglycemia

13. adverse event and disease or the like 
Observation throughout observation period

point

Observation Classification, outcome, severity, relationship, etc. of adverse event and disease or the item like.

Adverse event and disease or the like are observed throughout the study. Adverse event and disease or the like include the side effects of the medication and clinically significant abnormal fluctuations in test results. Investigators collect the information of presence/absence of hypoglycemia, hypoglycemia, other adverse events and disease or the like by interview at every observation point. The occurrence of the adverse events and disease or the like is recorded onto the carte and CRF. The study subjects are further followed up if necessary.

*The classification of adverse events and disease or the like is based on MedDRA/J. Refer "10. Safety evaluation analysis"

Table 4. Observation schedule 


\begin{tabular}{|c|c|c|c|c|c|c|}
\hline \multirow[t]{3}{*}{ observation item } & \multicolumn{6}{|c|}{ observation period / observation point } \\
\hline & \multirow[t]{2}{*}{ enrollment } & week & \multirow{2}{*}{$\begin{array}{l}\text { baseline } \\
\text { week } 0^{*}\end{array}$} & \multirow{2}{*}{$\begin{array}{l}\text { week } \\
2 \\
\pm 1 \\
\text { week }\end{array}$} & \multirow{2}{*}{$\begin{array}{l}\text { week } \\
4 \\
\pm 1 \\
\text { week }\end{array}$} & \multirow{2}{*}{$\begin{array}{l}\text { at } \\
\text { disconti- } \\
\text { nuation }\end{array}$} \\
\hline & & $\mathbb{8 4 *}$ & & & & \\
\hline obtaining consent & 0 & & & & & \\
\hline \Eligibility information & - & & & & & \\
\hline$\triangle$ Background information & - & $\bullet$ & & & & \\
\hline 『physical examination & & ○ & ○ & $\triangle$ & ○ & $\triangle$ \\
\hline $\begin{array}{l}\text { \medication information (except } \\
\text { study agent and insulin) }\end{array}$ & & ○ & ○ & $\triangle$ & ○ & $\triangle$ \\
\hline $\begin{array}{l}\text { 囚medication information (study agent, } \\
\text { insulin) }\end{array}$ & & $\leftarrow$ & $\rightarrow$ & & & \\
\hline 冈blood tests & & 0 & ○ & $\triangle$ & ○ & $\triangle$ \\
\hline \urine tests & & ○ & ○ & $\triangle$ & - & $\triangle$ \\
\hline \special blood tests & & $\boldsymbol{\Delta}$ & $\Delta$ & & $\Delta$ & $\Delta$ \\
\hline 囚UCG & & ○ & & & ○ & $\triangle$ \\
\hline 『QOL score & & ○ & - & & ○ & \\
\hline \multirow{2}{*}{$\begin{array}{l}\Downarrow \text { Other items the study subjects } \\
\text { measure by themselves } 1\end{array}$} & & \multicolumn{2}{|c|}{28 days } & \multicolumn{2}{|c|}{28 days } & \\
\hline & & प & & प & & \\
\hline $\begin{array}{l}\bowtie \text { Other items the study subjects } \\
\text { measure by themselves } 2\end{array}$ & & $\leftarrow$ & $\rightarrow$ & & & \\
\hline \adverse event and disease or the like & & $\leftarrow$ & $\rightarrow$ & & & \\
\hline
\end{tabular}

required

$\triangle$ optional

$\Delta$ conduct if residual sample exist

${ }^{*}$ conduct before the start of the study agent administration

\section{Figures}




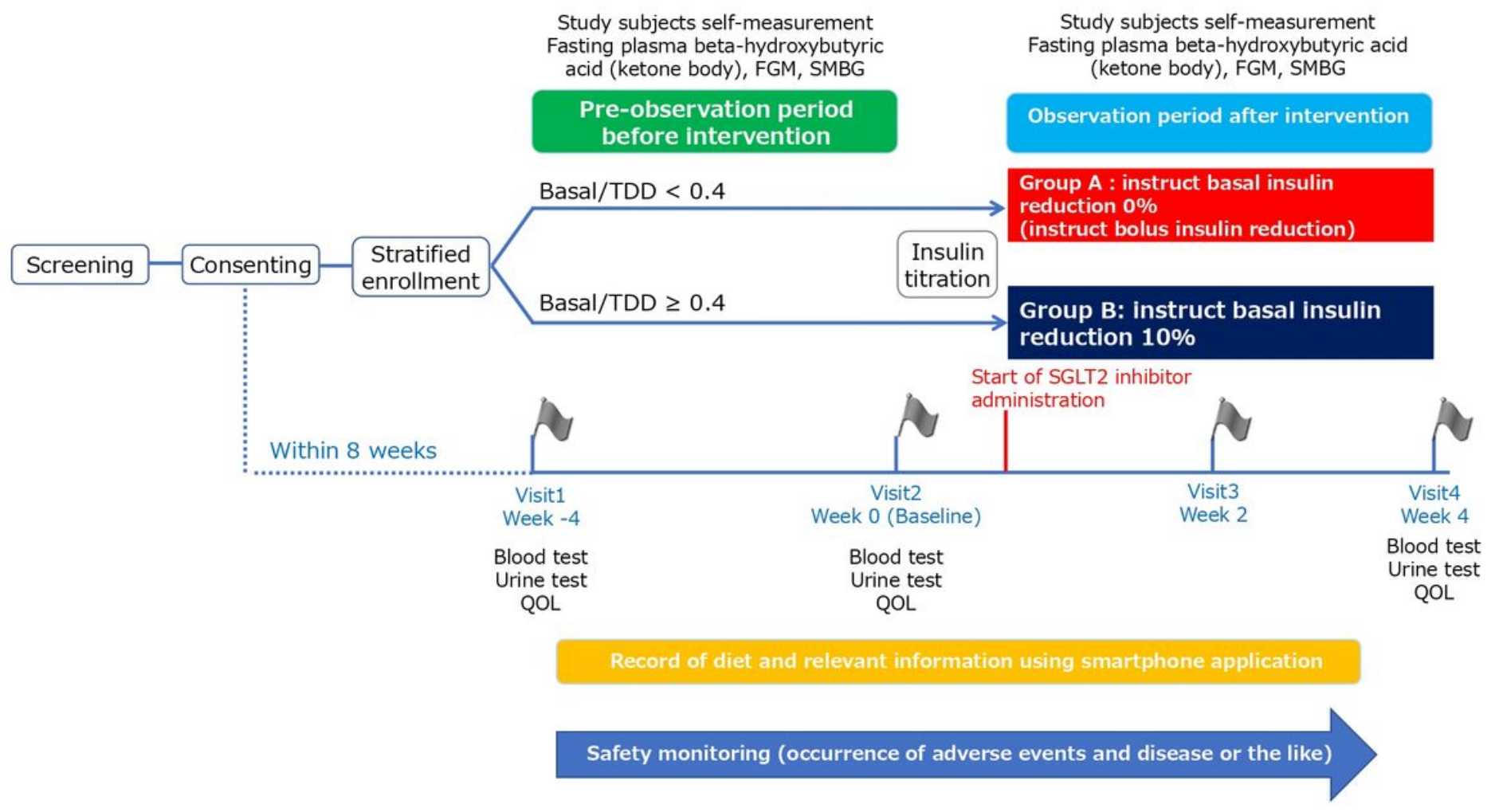

\section{Figure 1}

The study design The study subjects are stratified into two groups based on the ratio of basal insulin (Basal) to the total daily insulin dose (TDD) (Basal/TDD, $<0.4$ or $>0.4$ ). The study does not involve randomization of participants. 


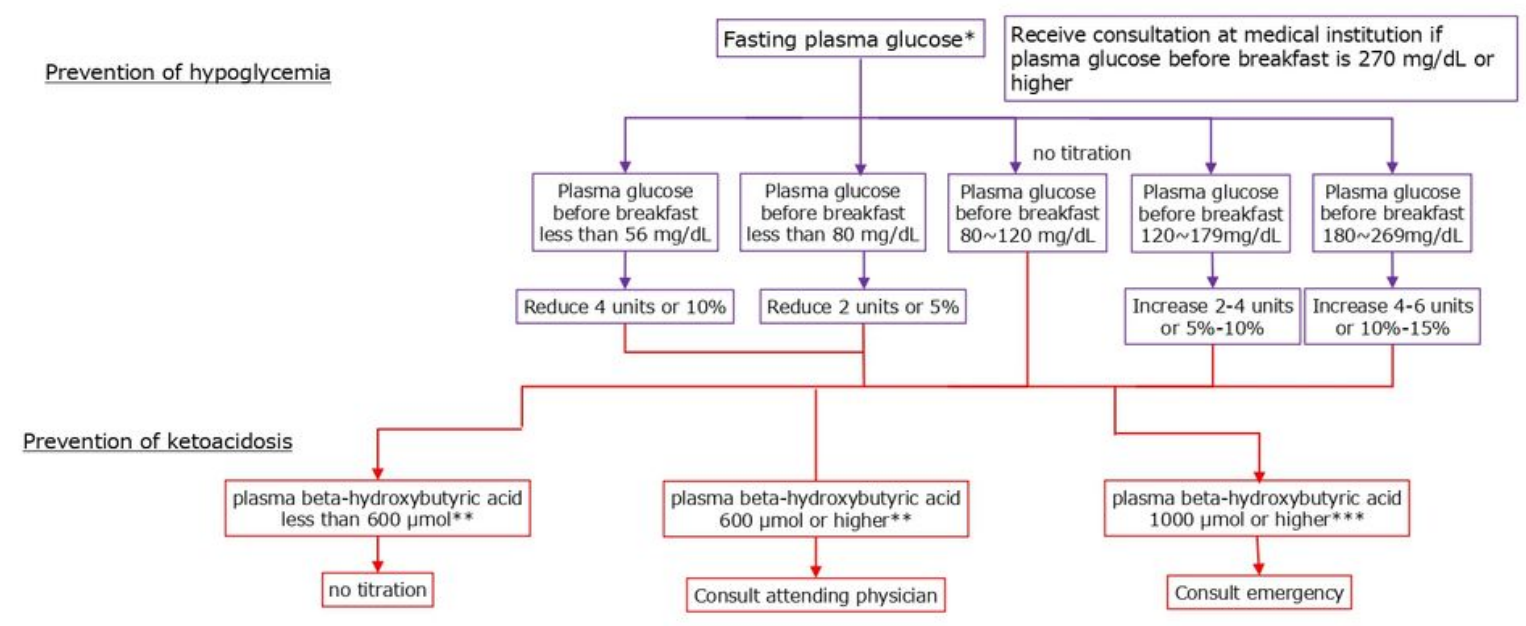

\section{Figure 2}

Algorism for Basal Insulin Titration after SGLT2 administration The study subjects are to follow the dosing instruction for 3 days from the start of the intervention after which the basal and bolus insulin can be titrated either by the subject or by instruction from the attending physician according to "Algorithm for Basal Insulin Titration after SGLT2 administration." 


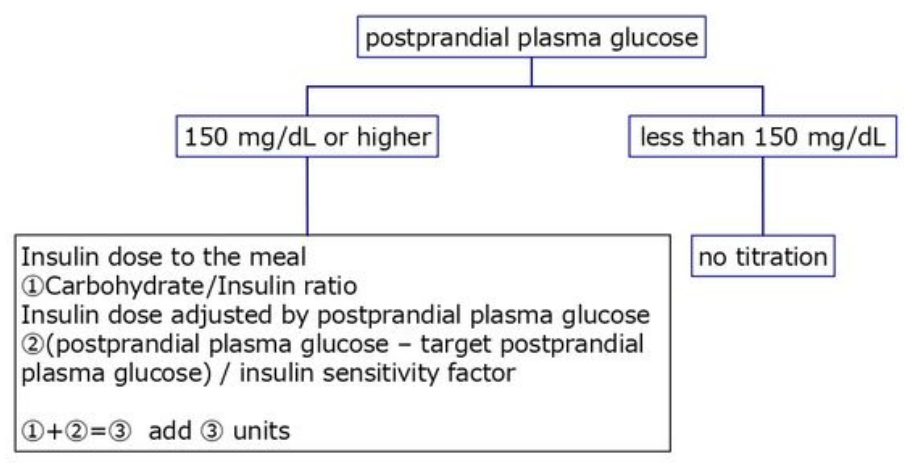

\section{Figure 3}

Algorism for Bolus Insulin Titration after SGLT2 administration The study subjects are to follow the dosing instruction for 3 days from the start of the intervention after which the basal and bolus insulin could be titrated either by the subject or by instruction from the attending physician according to "Algorithm for Bolus Insulin Titration after SGLT2 administration 


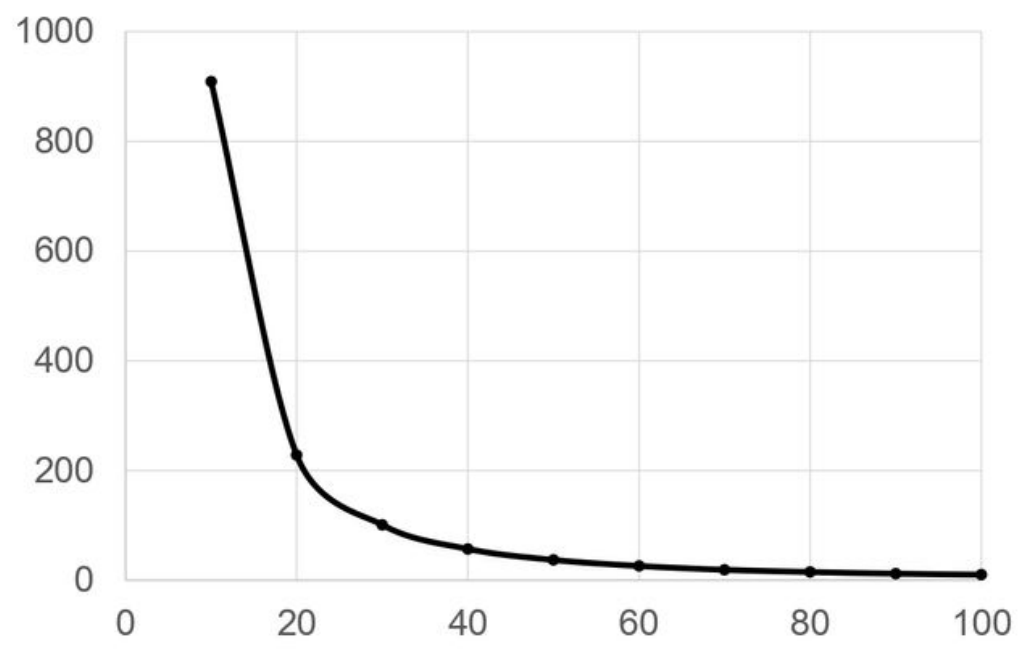

\section{Figure 4}

The assumed increase of hypoglycemia and required sample size The baseline hypoglycemia was set as $7 \pm 6$ times/month. Increase of hypoglycemia is expressed as \% increase from the baseline (shown in $X$ axis). The minimum sample size required to achieve a significance of 0.05 for a one-sided t-test with a statistical power at $80 \%$ is determined. The necessary sample size is expressed as number of patients in one arm (shown in Y axis). When sample size is 29 patients in one group, \% increase of the hypoglycemia was more than $60 \%$, the sample size is estimated to be enough.

\section{Supplementary Files}

This is a list of supplementary files associated with this preprint. Click to download.

- renamed43b8f.pdf 